\title{
Parâmetros populacionais para Triatoma sordida Stal, 1859, o vetor mais freqüente da doença de Chagas no Triângulo Mineiro (Heteroptera, Triatominae)
}

\author{
Population parameters for Triatoma sordida Stal, 1859: the most frequent vector \\ for Chagas disease in the Triângulo Mineiro (Heteroptera, Triatominae)
}

\author{
Afonso Pelli ${ }^{1}$, Michel Alves da Silva ${ }^{1}$, Fabiano Rodrigues Sarmento ${ }^{2}$, Elizabeth Martins ${ }^{1}$, \\ Simone Alves da Mata ${ }^{3}$, Marcos Abel Domingues ${ }^{3}$ e Luis Eduardo Ramirez ${ }^{1}$
}

\begin{abstract}
RESUMO
O Triatoma sordida é o mais freqüente vetor do Trypanosoma cruzi, Chagas, 1909, em Uberaba, MG. O objetivo deste trabalho foi construir uma tabela de vida dinâmica para o Triatoma sordida visando fornecer dados para subsidiar o controle de suas populações.
\end{abstract}

Palavras-chaves: Triatoma sordida. Tabela de vida dinâmica Parâmetros populacionais.

\begin{abstract}
Triatoma sordida is the most frequent vector for Trypanosoma cruzi, Chagas, 1909, in Uberaba, State of Minas Gerais. The objective of this study was to construct a dynamic life table for Triatoma sordida with the aim of supplying support data for controlling its populations.
\end{abstract}

Key-words: Triatoma sordida. Dynamic life table. Population parameters.

Desde o início da década de 1970, tabelas de vida vêm sendo obtidas para vários triatomíneos ${ }^{3} 9$ 16, sendo algumas características populacionais determinadas. 0 Triatoma sordida apresenta importantes características que fazem dele um possível vetor, como a capacidade de colonizar o peridomicílio ${ }^{7}$.

Segundo a Secretaria Municipal de Saúde de Uberaba, neste município, Triatoma sordida corresponde a $85 \%$ dos triatomíneos capturados no campo, entre janeiro de 1995 e maio de 2004, sendo que $86 \%$ dos barbeiros positivos para Trypanosoma cruzi, foram dessa espécie.

A tabela de vida dinâmica ou de coorte é uma abordagem, apontada por vários autores, para conhecer as estratégias reprodutivas e história de vida das populações ${ }^{7131416}$, fornecendo informações sobre as relações das populações com o meio.

0 objetivo deste trabalho foi construir uma tabela de vida dinâmica para Triatoma sordida, visando fornecer dados para subsidiar o controle de suas populações.

\section{MATERIAL E MÉTODOS}

Os animais foram criados em um insetário que possui controle de temperatura e umidade. Os valores médios de temperatura e umidade relativa foram $28^{\circ} \mathrm{C} \pm 2,1^{\circ} \mathrm{C}$ e $68 \% \pm 11,1 \%$, respectivamente.

Os insetos foram capturados na região de Itapagipe e Iraí de Minas (localizados no Triângulo Mineiro e Alto Paranaíba) em setembro de 1998, e foram doados pela Fundação Nacional de Saúde ao Departamento de Parasitologia da Universidade Federal do Triângulo Mineiro.

Os frascos de Borrel, onde foram criados os Triatoma sordida, são munidos com suporte de cartolina e fechados. A alimentação dos insetos foi realizada por meio de repastos semanais em galinhas (Gallus domesticus).

As contagens foram efetuadas semanalmente e o material descartável, sejam eles ovos, mudas ou mortos, acondicionados em frasco contendo álcool, em solução aquosa de $75 \%$.

\footnotetext{
1 Departamento de Ciências Biológicas, Universidade Federal do Triângulo Mineiro, Uberaba, MG. 2. Delegacia de Polícia Civil de Uberaba, Uberaba, MG. 3. Seção de Zoonoses da Secretaria Municipal de Saúde de Uberaba, Uberaba, MG.

Endereço para correspondência: Dr. Afonso Pelli. Dept to de Ciências Biológicas/UFTM. R. Frei Paulino 30, 38025-180 Uberaba, MG.

e-mail: apelli.oikos@dcb.uftm.edu.br

Recebido para publicação em 8/9/2005

Aceito em 12/1/2007
} 
A fecundidade das fêmeas, expressa em progênies produzidas por estação de reprodução ou faixa etária, é designada por b . Foi obtida pelo número total de ovos recolhidos dividido pelo total de fêmeas adultas da coorte. A tabela de vida retrata a estatística de mortalidade em diversos tempos $\left(m_{x}\right)$, bem como a probabilidade de sobrevivência $\left(S_{x}\right)$ entre as idades $\mathrm{x}$ e $\mathrm{x}+1$. As probabilidades de sobrevivência sobre muitos intervalos de idade são resumidas pela supervivência à idade $\mathrm{x}$ (1), que é a probabilidade de que um indivíduo recém-nascido estar vivo na idade $x$. Uma medida adicional incluída na tabela de vida é a expectativa de vida $\left(\mathrm{e}_{\mathrm{x}}\right)$ de um indivíduo de idade $\mathrm{x}^{17}$.

A taxa reprodutiva líquida ou taxa líquida de reprodução, que é o número de fêmeas produzido por uma fêmea, pode ser indicada por $\mathrm{R}_{0}$; sendo calculada pelo somatório do produto das colunas supervivência e fecundidade específicas por idade $\left(\sum \mathrm{l}_{\mathrm{x}} \mathrm{b}_{\mathrm{x}}\right)$. 0 tempo médio de geração, designado pelos demógrafos como G ou T, ou seja, o tempo médio entre duas gerações sucessivas; ou o período médio entre nascimento dos progenitores e da prole, é dado pelo somatório do produto das colunas $\mathrm{x}, \mathrm{1}_{\mathrm{x}}, \mathrm{e} \mathrm{b}_{\mathrm{x}}$, dividido por $\mathrm{R}_{0}$.

A taxa reprodutiva instantânea diária, indicada por " $\mathrm{r}$ " foi calculada com base na equação: $\log \left(\mathrm{R}_{0}\right) / \mathrm{G}$.

\section{RESULTADOS E DISCUSSÃO}

A tabela de vida apresenta a sobrevivência e fecundidade do Triatoma sordida sob condições experimentais durante 402 dias. A idade está representada em número de dias do intervalo de x ax+1 e o tempo foi tabulado em intervalos de aproximadamente 51 dias.

Conforme apresentado na Tabela 1 , dos 40 animais vivos no $t_{0}$ (eclosão dos ovos), 22 estavam vivos aos 52 dias ( $\left.\mathrm{t}_{1}\right)$. Disto $\mathrm{S}_{0}$ e $\mathrm{l}_{1}$, são ambos iguais a 0,55. A curva de sobrevivência é apresentada na Figura 1.

Tabela 1 - Tabela de vida dinâmica para Triatoma sordida, criado em condições de laboratório.

\begin{tabular}{lrrrrrr}
\hline $\mathrm{t}_{\mathrm{x}}$ & $\mathrm{n}_{\mathrm{x}}$ & $\mathrm{l}_{\mathrm{x}}$ & $\mathrm{m}_{\mathrm{x}}$ & $\mathrm{S}_{\mathrm{x}}$ & $\mathrm{e}_{\mathrm{x}}$ & $\mathrm{b}_{\mathrm{x}}$ \\
\hline $\mathrm{t} 0$ & 40 & 1,000 & 0,450 & 0,550 & 2,88 & \\
$\mathrm{t} 1$ & 22 & 0,550 & 0,091 & 0,909 & 3,82 & \\
$\mathrm{t} 2$ & 20 & 0,500 & 0,100 & 0,900 & 3,15 & \\
$\mathrm{t} 3$ & 18 & 0,450 & 0,056 & 0,944 & 2,44 & \\
$\mathrm{t} 4$ & 17 & 0,425 & 0,294 & 0,706 & 1,56 & 40,2 \\
$\mathrm{t} 5$ & 12 & 0,300 & 0,583 & 0,417 & 1,00 & 80,5 \\
$\mathrm{t} 6$ & 5 & 0,125 & 0,600 & 0,400 & 0,70 & 103,7 \\
$\mathrm{t} 7$ & 2 & 0,050 & & & & 16,5 \\
\hline
\end{tabular}

$\mathrm{t}$ - intervalo de tempo, $\mathrm{n}$ - número de indivíduos vivos, 1 - supervivência, $\mathrm{m}$ - taxa de mortalidade, $S_{x}$ - taxa de sobrevivência, $e_{x}$ - expectativa de vida, $b_{x}$ - fecundidade.

A curva de sobrevivência, construída com os dados de supervivência, pode apresentar-se de diversas formas. Como observado em alguns hidrozoários, a taxa de mortalidade pode ser maior nos estágios iniciais de vida e manter-se constante em baixos níveis, até a senescência ou morte programada ${ }^{8}$. Em vários mamíferos, observa-se baixa taxa de mortalidade, propiciando então ao menos um episódio reprodutivo para a maior parte dos indivíduos da populaçãa $0^{81718}$. Os dados obtidos indicam um padrão intermediário para o Triatoma sordida; neste

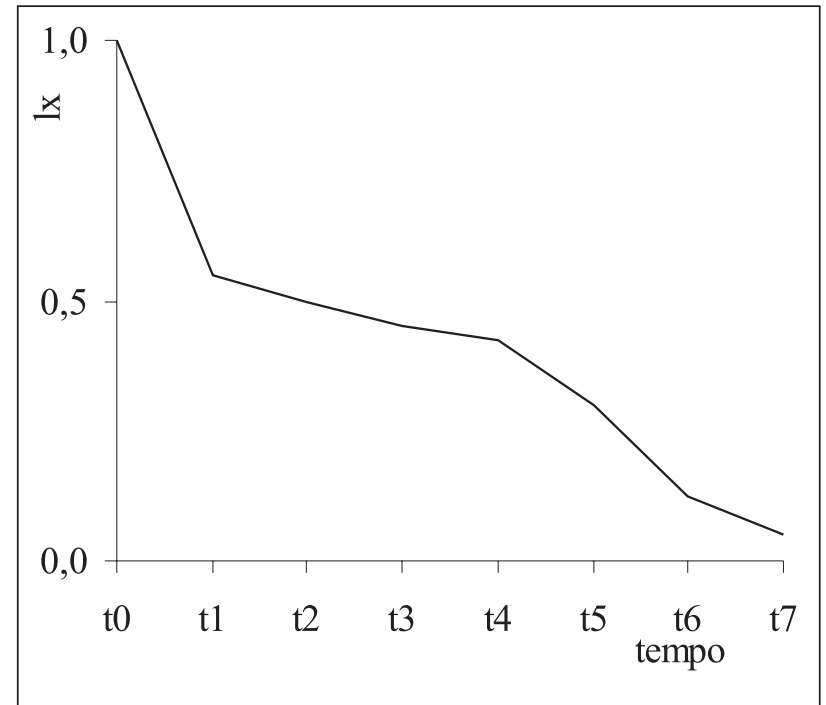

Figura 1 - Curva de sobrevivência para o Triatoma sordida, criado em condições de laboratório.

caso observou-se uma taxa de mortalidade inicial elevada, com posterior redução. Os dados indicam que aproximadamente $50 \%$ da população atinge a idade reprodutiva.

Em trabalho semelhante, observou-se que Triatoma sordida pode apresentar sobrevivência relativamente constante ao longo do desenvolvimento da coorte ${ }^{15}$. Este padrão não foi observado no presente estudo, porém a curva de sobrevivência observada se aproxima do Tipo $\mathrm{II}^{8}$, sendo a taxa de mortalidade relativamente constante, apresentando dois picos, o primeiro no $\mathrm{t}_{0} \mathrm{e}$ o segundo no $\mathrm{t}_{5} \mathrm{et}_{6}$.

O esperado seria que a maior parte de uma população natural fosse constituída por jovens, o que é constatado no campo, com os dados coletados pela Secretaria Municipal de Saúde de Uberaba. Neste caso, a mortalidade é afetada por mudanças ontogenéticas, estando a mortalidade intimamente associada aos períodos de muda.

Os indivíduos começaram a chegar à fase adulta aos $183 \pm 36$ dias e, após 15 dias começam a ovipor. Em $\mathrm{t}_{4}$ a taxa observada foi de 40,2 ovos por fêmea, no $\mathrm{t}_{5} 80,5$, em t 6 103,7 e em t a taxa foi de 16,5 ovos por fêmea; produzindo, em média, um total de 240,9 ovos/fêmea. Soares observou que T. pseudomaculata produz 264 e T. brasiliensis 142 ovos/fêmea, respectivamente.

0 valor obtido para o tempo médio de geração foi 4,955, indicando intervalo de aproximadamente 253 dias entre gerações, já que os intervalos de tempo foram tabelados a cada 51 dias.

Ponderando o número de ovos produzidos, Triatoma sordida, no nosso estudo, produziu, relativamente um grande número de ovos. Porém, quando comparado com outras espécies, 0 valor da taxa reprodutiva instantânea diária $\left[\mathrm{r}=\log \left(\mathrm{R}_{0}\right) / \mathrm{G}\right]$ obtida foi relativamente baixa, sendo igual a 0,007 , enquanto T. brasiliensis apresenta 0,010; T. pseudomaculata, 0,009 e Triatoma sordida com $0,012^{15}$.

Quando Triatoma sordida é alimentado com pombos e cobáios, o tempo desde a eclosão dos ovos até a morte do último 
indivíduo varia de 420 a 659 dias $^{4}$. Em comparação com outros triatomíneos o Triatoma sordida apresenta elevada expectativa de vida. No presente estudo o observado foi de $280 \pm 53$, com valor máximo de 402 dias, o que pode ser explicado, provavelmente, por se tratarem de populações distintas, com diferentes histórias de vida. Sabe-se que uma população apresenta continuidade temporal e espacial, por causa dos descendentes, herdando genes de reservatório comum. A história adaptativa compartilhada pelos indivíduos que constituem uma população representa um importante atributo desta unidade ecológica. Também devem ser ponderadas diferenças quanto ao ambiente físico, já que diferenças ambientais podem influenciar diversos atributos populacionais ${ }^{3}$.

Fatores do ambiente, incluindo as condições físicas e alimentação podem influenciar parâmetros populacionais. Rhodnius neglectus apresenta ciclo de vida mais curto quando se alimenta em mamíferos, quando comparado com fonte alimentar em aves ${ }^{5}$. Mudanças no ciclo de vida de Triatoma klugi, em função da fonte alimentar, também já foram relatadas-camundongos ou galinhas'; assim como mudanças significativas para Meccus picturatus, quando alimentados com galinhas ou coelhos ${ }^{10}$.

A taxa reprodutiva líquida, que é o número de fêmeas produzido por uma fêmea, apresentou valor de $\mathrm{R}_{0}$ igual a 55 fêmeas/geração.

Ponderando o modelo de crescimento geométrico, nota-se que, através da projeção da população, apresentada na tabela de vida dinâmica, observa-se que no intervalo de tempo $t=229$; 0 valor de $\lambda$ estabiliza em 2,00546. Neste caso, $\lambda$ representa 0 valor de uma razão de acréscimo, ou decréscimo, da população, desde que as estimativas populacionais sejam separadas pela mesma quantidade de eventos de nascimentos e mortes.

Quando o valor de $\lambda$ apresenta-se estável, significa dizer que a população alcançou a distribuição etária estável; ou seja, as proporções etárias da população permanecem constantes ao longo de vários intervalos de tempo. Esta situação somente seria viável quando as taxas específicas, por classe etária, de mortalidade e natalidade, permanecessem constantes. Para que essa situação seja alcançada, seria necessário que as condições ambientais permanecessem constantes, por vários intervalos de tempo.

Quando atingisse a distribuição etária estável, uma população de Triatoma sordida seria composta por $67 \%$ de indivíduos na classe etária $0 ; 18,4 \%$ na classe etária $1 ; 8,3 \%$ na $2 ; 3,7 \%$ na $3 ; 1,7 \%$ na 4 ; $0,6 \%$ na $5 ; 0,1 \%$ na 6 e $0,03 \%$ na classe etária 7 . Distribuição etária em trabalho semelhante foi apontada para Triatoma sordida ${ }^{15}$.

A capacidade inata para crescer ou taxa intrínseca de aumento, em números, de uma população pode ser indicada por $r_{\mathrm{m}}$. Esta seria a taxa exponencial ou geométrica de aumento, quando a população apresentasse distribuição etária estável. Como os parâmetros ambientais mudam continuamente, tanto temporal, quanto espacialmente, raramente as populações alcançam à distribuição etária estável. 0 valor aproximado de $\mathrm{r}_{\mathrm{m}}$, pode ser designado por $\mathrm{r}$, que é uma aproximação da taxa intrínseca de aumento. 0 valor calculado foi igual a 0,809 . A partir do valor obtido de $r_{\mathfrak{a}}$, pode-se calcular o valor de lâmbida $(\lambda)$, obtendose 2,245 , o que é uma razoável aproximação do valor calculado $(2,00546)$; já que $r=\log _{e} \lambda$.
Pelo modelo de crescimento geométrico o tempo necessário para dobrar o tamanho da população, designado aqui por $\mathrm{t}_{2}$, seria $\log _{e \lambda}$, isto é: $0,69 / \log _{\mathrm{e}} \lambda ;$ o que corresponde a 0,85 ano ou 310 dias.

Estudos morfométricos deveriam ser realizados visando determinar a ocorrência de possíveis diferenças quantitativas entre populações ${ }^{11}{ }^{12}$, o que poderia indicar que a distribuição geográfica pode gerar, em diferentes ambientes, populações adaptadas às condições ambientais diversas, inclusive à colonização de ambientes domésticos.

Buscando racionalizar as operações de controle dos vetores de T. cruzi, é fundamental que se conheça a dinâmica populacional de cada uma das espécies envolvidas ${ }^{19}$. Questões como atividade sazonal, razão sexual, mobilidade ${ }^{13}$, resposta comportamental a inseticidas ${ }^{12}$, tempo de defecação após repasto, em função da fonte alimentar 10111213 devem ser esclarecidas, visando assim criar condições favoráveis a erradicação dos vetores de uma das moléstias que produz o maior ônus de enfermidade entre as denominadas doenças tropicais.

\section{REFERÊNCIAS}

1. Alzogaray RA, Zerba EM. Behavioral response of fifth instar nymphs of Triatoma infestans (Hemiptera: Reduviidae) to pyrethroids. Acta Tropica 78: 51-57, 2001.

2. Botto-Mahan C, Cattan PE, Canals M. Field tests of carbon dioxide and conspecifics as baits for Mepraia spinolai, wild vector of Chagas disease. Acta Tropica 82: 377-380, 2002.

3. Cabello DR. Effects of environmental temperature on life tables of Rhodnius neivai Lent, 1953 (Hemiptera: Reduviidae) under experimental conditions. Memórias do Instituto Oswaldo Cruz 94: 709-714, 1999.

4. Dias E. Notas sobre o tempo de evolução de algumas espécies de triatomíneos em laboratório. Revista Brasileira de Biologia 15: 157-158, 1955.

5. Diotaiuti L, Dias JCP. Estudo comparativo do ciclo evolutivo de Rhodnius neglectus alimentados em pombos ou camundongos. Revista da Sociedade Brasileira de Medicina Tropical 20: 95-100, 1987.

6. Diotaiuti L, Penido CM, Pires HHR, Dias JCP. Dinâmica da alimentação e dejeção do Triatoma infestans. Revista da Sociedade Brasileira de Medicina Tropical 28: 195-198, 1995.

7. Emmanuelle-Machado P, Koerich LB, Joukoski DB, Carvalho-Pinto CJ, Grisard EC, Steindel M. Biology of Triatoma klugi Carcavallo, Jurberg, Lent \& Galvão 2001 (Heteroptera: Reduviidae) under laboratory conditions: effects of distinct blood sources and susceptibility to Trypanosoma cruzi and Trypanosoma rangeli. Memórias do Instituto Oswaldo Cruz 97: 583-587, 2002.

8. Hutchinson GE. Introdução a la Ecologia de poblaciones. Blusse Milanesado, Barcelona, 1981.

9. Juarez E, Silva E. Comportamento do Triatoma sordida em condições de laboratório. Revista Saúde Pública 16: 1-36, 1982.

10. Martínez-Ibarra JA., Grant-Guillén Y, Martínez-Grant DM. Feeding, defecation, and development times of Meccus longipennis Usinger, 1939 (Hemiptera: Reduviidae: Triatominae) under laboratory conditions. Memórias do Instituto Oswaldo Cruz 98: 899-903, 2003a.

11. Martínez-Ibarra JA, López MN, Robles MRH, Guillén YG. Influence of the Blood Meal Source on the Biology of Meccus picturatus Usinger 1939 (Hemiptera: Reduviidae: Triatominae) under Laboratory Conditions. Memórias do Instituto Oswaldo Cruz 98: 227-232, 2003b.

12. Monroy C, Bustamante DM, Rodas A, Rosales R, Mejía M, Tabaru Y. Geographic distribution and morphometric differentiation of Triatoma nitida Usinger 1939 (Hemiptera: Reduviidae: Triatominae) in Guatemala. Memórias do Instituto Oswaldo Cruz 98: 37-43, 2003. 
13. Monroy C, Marroquin R, Rodas A, Rosales R, Jaenson TGT. Dispersion and colonization of Triatoma ryckmani (Hemiptera: Reduviidae) in artificial environments in a semiarid region of a Chagas disease endemic area in Guatemala. Acta Tropica 91: 145-151, 2004

14. Moss R, Watson A, Ollason J. Animal Population Dynamics. Chapman and Hall, Washington, 1982.

15. Oscherov EB, Damborsky MP, Bar ME, Porcel E. Estadísticos poblacionales de Triatoma sordida Stäl 1859 (Hemiptera: Reduviidae) en condiciones experimentales. Revista de Saúde Pública 30: 438-443, 1996.
16. Rabinovich JE. Vital statistics of Triatominae (Hemiptera: Reduviidae) under laboratory conditions. I. Triatoma sordida Klug. Journal of Medical Entomology 9: 351-370, 1972.

17. Rabinovich JE. Ecologia de poblaciones animales. Eva V. Chesneau, Washington, 1978

18. Ricklefs RE. Economia da Natureza. Guanabara Koogan, Rio de Janeiro, 2003.

19. Silveira AC, Costa JR. Doença de Chagas e controle do vetor: desafios atuais, habitacionais e ambientais. Revista de Patologia Tropical 29: 199-212, 2000 . 